\title{
Un estudio comparativo entre dos herramientas de respuesta de audiencia en las aulas universitarias*
}

\author{
ILU VALLET-BELLMUNT** \\ TERESA VALLET-BELLMUNT***

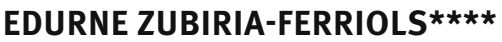 \\ TERESA MARTÍNEZ-FERNÁNDEZ***** \\ INMACULADA BEL-OMS******
}

* Agradecemos la financiación prestada por la Unitat de Suport Educatiu de la UJI, concretamente a los proyectos: 3117/15: “Aprenentatge cooperatiu: formació d’equips i avaluació entre iguals. Una comparació multidisciplinaria i internacional”; 3485/17: "GACETEAM. Google Apps for Cooperative Education: Team Work".

** Doctoranda en Marketing. Profesora Asociada. Universitat Jaume I, Castellón, España. E-mail: mvallet@uji.es. ORCID: oooo0002-7362-4119. Google Scholar: https://scholar.google.com/citations?hl=es\&user=aE_qPLUAAAAJ.

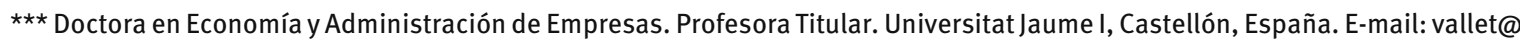
uji.es. ORCID: 0000-0003-0477-0101. Google Scholar: https://scholar.google.com/citations?hl=es\&user=j5SVLVgAAAJ.

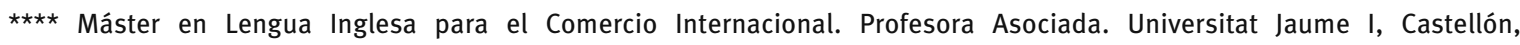
España. E-mail: zubiria@uji.es. ORCID: 0000-0002-9754-7891. Google Scholar: https://scholar.google.es/ citations?hl=ca\&user=QwQMLbEAAAAJ.

$\star * \star \star *$ Doctora en Dirección de Empresas. Profesora Titular. Universitat Jaume I, Castellón, España. E-mail: tmartine@uji.es. ORCID: 0000-0003-0556-5409. Google Scholar: https://scholar.google.com/ citations?hl=es\&amp;user=kGIb010AAAAJ\&user=kGIb010AAAAJ.

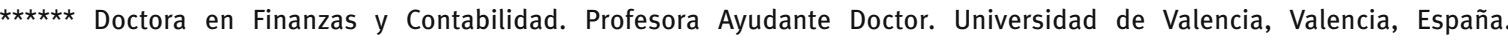
E-mail: inmaculada.bel@uv.es. ORCID: 0000-0001-7148-0652. Google Scholar: https://scholar.google.es/ citations?hl=ca\&user=ozauiGIAAAAJ. 


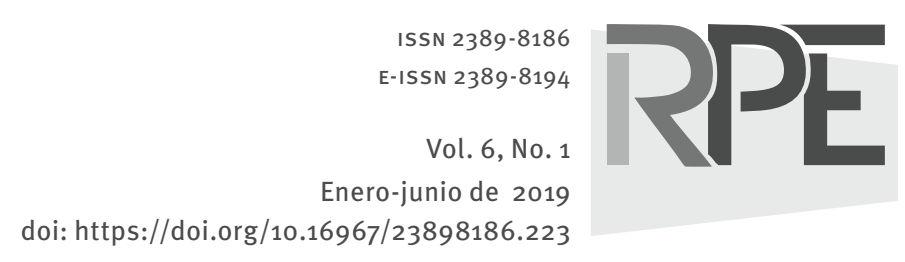

COMO CITAR ESTE ARTÍCULO How to cite this article:

Vallet-Bellmunt, I. et al. (2019). Un estudio comparativo entre dos herramientas de respuesta de audiencia en las aulas universitarias. Revista Perspectiva Empresarial, 6(1), 61-73

Recibido: 14 de junio de 2018 Aprobado: 22 de octubre de 2018
RESUMEN El objetivo de este texto es investigar la influencia del uso de herramientas tecnológicas en las aulas universitarias. En concreto, analizamos y evaluamos la aplicación de dos herramientas de respuesta de audiencia libres (específicamente Socrative y Kahoot!) como herramientas que fomentan la participación y asistencia de los alumnos a las sesiones teóricas y facilitan el proceso de enseñanza-aprendizaje. Se analiza si estas herramientas pueden ser utilizadas como motivadoras, fomentadoras de la participación del alumnado y facilitadoras del aprendizaje. Además, la opinión de los estudiantes sobre la utilidad de estas herramientas en el aula y como métodos de enseñanza-aprendizaje. La muestra de este estudio está compuesta por 60 estudiantes de una universidad pública española en el Grado en Publicidad y Relaciones Públicas y Grado en Comunicación Audiovisual. Esta investigación es innovadora en el área de las TIC y tiene implicaciones para los profesores universitarios debido a la relevancia de la utilización de las nuevas tecnologías en las aulas con la finalidad de atraer la participación y asistencia de los alumnos y al mismo tiempo motivarlos a estudiar y a aprender.

PALABRAS CLAVE mobile learning, innovación, Kahoot!, Socrative, mejora académica, rendimiento académico, participación estudiantil.

\section{A comparative study between two audience response tools in university classrooms}

ABSTRACT The aim of this paper is to investigate the influence of the use of technological tools in university classrooms. In particular, we analyze and evaluate the application of two free audience-response tools (specifically, Socrative and Kahoot!) as tools that foster students' participation in and attendance to the theoretical sessions and facilitate the teaching-learning process. It is analyzed whether these tools can be used to motivate and foster participation and to facilitate learning. Furthermore, students' opinion on the usefulness of these tools in the classroom and as teaching-learning methods is also analyzed. The study sample is made up of 60 students at a Spanish public university in the Publicity and Public Relations and Audiovisual Communication programs. This research is innovative in the area of ICT and has implication for university teachers due to the relevance of the use of new technologies in the classroom with the aim of attracting students' participation and attendance while at the same time motivating them to study and learn.

KEY WORDS Mobile learning, innovation, Kahoot!, Socrative, academic improvement, academic performance, student participation. 


\section{Um estudo comparativo entre duas ferramentas de resposta de audiência nas aulas universitárias}

RESUMO O objetivo deste texto é investigar a influência do uso de ferramentas tecnológicas nas salas universitárias. Em concreto, analisamos e avaliamos a aplicação de duas ferramentas de resposta de audiência livres (especificamente Socrative e Kahoot!) como ferramentas que fomentam a participação e assistência dos alunos às sessões teóricas e facilitam o processo de ensino-aprendizagem. Se analisa se estas ferramentas podem ser utilizadas como motivadoras, fomentadoras da participação dos alunos e facilitadoras da aprendizagem. Ademais, a opinião dos estudantes sobre a utilidade destas ferramentas na sala e como métodos de ensino-aprendizagem. A amostra deste estudo está composta por 60 estudantes de uma universidade pública espanhola na Graduação em Publicidade e Relações Públicas e Graduação em Comunicação Audiovisual. Esta investigação é inovadora na área das TIC e tem implicações para os professores universitários devido à relevância da utilização das novas tecnologias nas salas de aula com a finalidade de atrair a participação e assistência dos alunos e ao mesmo tempo motivá-los a estudar e a aprender.

PALAVRAS-CHAVE mobile learning, inovação, Kahoot!, Socrative, melhora acadêmica, rendimento acadêmico, participação estudantil. 


\section{Introducción}

La globalización, la revolución de Internet, la mejora de las conexiones inalámbricas, la evolución de las tecnologías de la información, los teléfonos inteligentes, tabletas y otras herramientas tecnológicas son parte de los cambios que se están produciendo en la sociedad de hoy. Esto ha supuesto también un cambio para los contextos de aprendizaje, configurando nuevas oportunidades y retos (Masrom and Ismail, 2010). Las instituciones educativas en general, y los centros de educación superior en particular, no pueden mantenerse al margen de estos cambios; por ello deben ser más flexibles y deben facilitar al máximo el desarrollo de las potencialidades de su alumnado.

La evolución imparable de la tecnología hace que a finales de la década de los 90 empiece a hablarse de aprendizaje electrónico o enseñanza a través de medios informáticos. El aprendizaje electrónico (e-learning) se identifica como el proceso de enseñanza y aprendizaje a través de medios electrónicos. En sus inicios este tipo de enseñanza estaba principalmente relacionada con el aprendizaje a distancia debido a su fuerte vínculo con la Web (Crompton, 2013). Suscitado por los avances tecnológicos -tanto en los dispositivos como en las comunicaciones inalámbricasse introduce la adaptación del modelo e-learning a dispositivos móviles, teléfonos, PDA, otros gadgets, y ahora tabletas, que permiten desarrollar el aprendizaje desde cualquier lugar. Este modelo pasa a denominarse Mobile learning (m-learning), generando un gran interés en la comunidad científica y educativa (Naismith et al., 2004; West, 2012; Ally and Prieto-Blázquez, 2014; Fundación Telefónica, 2014). El concepto m-learning conlleva el uso de la tecnología móvil como herramienta de aprendizaje dentro y fuera del aula en cualquier momento y en cualquier lugar (UNESCO, 2016). Estudios previos corroboran que la utilización del m-learning en el aula puede mejorar el proceso de aprendizaje (West, 2012; Hamdan and Amorri, 2016) dado que la utilización de aplicaciones en determinadas materias pueden mejorar el proceso de enseñanza-aprendizaje, la relación entre los alumnos y la relación alumnos-profesores (Mtebe and Raisamo, 2014; Vázquez-Cano, 2014). Esta premisa ha sido apoyada por diversos autores en diferentes niveles del proceso de enseñanza-aprendizaje, concretamente: en los centros de enseñanza obligatoria (Suñol et al., 2016), en la universidad (Brebera, 2016) o en diferentes áreas tales como la narrativa digital (Herrington, 2009), matemáticas (Suñol et al., 2016) o en el aprendizaje de lenguas extranjeras (Brebera, 2016). En la universidad, los móviles son utilizados como una herramienta esencial (Yu, 2012; Yu and Conway, 2012) puesto que ofrecen muchas posibilidades para los estudiantes como medios de comunicación y para el trabajo autónomo o el trabajo colaborativo (Humanante-Ramos and García Peñalvo, 2013). Sánchez-Prieto et al. (2014) los consideran un mecanismo que permite a los usuarios aprender en cualquier lugar y momento -aunque son muy difíciles de alcanzar en países como Nigeria, Sudáfrica, Chile o Filipinas-, siendo al mismo tiempo un elemento que reduce el coste de los estudios universitarios (West, 2012).

Dentro del m-learning hay que considerar la utilización de herramientas de respuesta de audiencia - HRA - cuyo objetivo se centra en el aprendizaje activo por parte del alumno mediante un dispositivo móvil. Estas herramientas ofrecen al alumnado oportunidades de participación, motivación, comunicación, trabajo autónomo o colaborativo (Bicen and Kocakoyun, 2018). PazAlbo Prieto (2014) documenta que el uso de las HRA permite una mayor implicación y más compromiso de los estudiantes en el proceso de enseñanza-aprendizaje. Algunas de ellas ofrecen la posibilidad de utilizar juegos en el aula. Este concepto se conoce como 'ludificación' (Kapp, 2012), permitiendo que una prueba cotidiana -como un test- se convierta en una actividad lúdica y dinámica para el alumnado mediante estrategias que pueden incluir diferentes respuestas con diversas valoraciones, además de un feedback inmediato.

Hemos considerado que la Universitat Jaume I de Castellón, ubicada en España, es un buen contexto para estudiar el uso de las nuevas tecnologías en las aulas universitarias por dos razones: en primer lugar, España es considerado uno de los países europeos donde más del $80 \%$ de los centros no universitarios tienen acceso a Internet a través de wifi (Fundación Telefónica, 2010). En segundo lugar, las escuelas y las universidades han estado inmersas en la implementación de ordenadores en las aulas desde 2014; en donde solo el $30 \%$ de los libros de texto están impresos. España, por tanto, representa un escenario óptimo para analizar las relaciones dinámicas entre 
el uso de las nuevas tecnologías y los resultados de los alumnos en las universidades españolas. Resultados que podrían extrapolarse en contextos similares a nivel internacional.

De este modo el objetivo de este trabajo es doble: (i) analizar por parte del profesorado dos HRA gratuitas (Socrative y Kahoot!) como herramientas motivadoras, fomentadoras de la participación del alumnado y facilitadoras del aprendizaje, utilizadas con el alumnado de la asignatura "Marketing en el punto de venta" de las titulaciones de Publicidad y Relaciones Públicas y Comunicación Audiovisual de la Universitat Jaume I de Castellón; (ii) examinar la opinión del estudiantado sobre la funcionalidad de esas herramientas tecnológicas. Con esto, se pretende ofrecer una perspectiva complementaria de $360^{\circ}$. Así, este trabajo evidencia que el m-learning desempeña un papel importante en el área de la educación y en el uso particular de las HRA.

El presente trabajo está estructurado de la siguiente manera. En un primer momento se describe el marco teórico en relación al concepto HRA. A continuación, se desarrolla la metodología utilizada en este estudio y se presentan los resultados obtenidos. Finalmente se muestran las principales conclusiones seguidas de las limitaciones inherentes a este estudio, al tiempo que se indican las posibles líneas futuras de investigación.

\section{El concepto de herramientas de respuesta de audiencia}

Hoy existe una gran variedad de HRA cuyo objetivo se centra en la obtención/recolección de respuestas a través de Internet mediante un dispositivo conectado ya sea móvil (smartphone, tablet entre otros) o no (PC). En el presente estudio se ha considerado el uso de dos HRA gratuitas, a saber: Socrative y Kahoot!

Socrative es una herramienta de evaluación educativa en entornos digitales que permite al profesorado conocer las respuestas del alumnado en tiempo real a través de dispositivos móviles y ordenadores. Socrative permite evaluar rápidamente al estudiantado a través de actividades preparadas o preguntas sobre el tema que se está impartiendo durante el curso o videoconferencia. Para su funcionamiento se requiere Internet y un smartphone o tableta.

Kahoot!, es una plataforma gratuita que permite la creación de cuestionarios de evaluación (disponible en App o versión Web). Es una herramienta por la cual el profesorado crea concursos en el aula para aprender o reforzar el aprendizaje y donde el alumnado son los concursantes. El colectivo de alumnos elige su alias o nombre de usuario y contesta una serie de preguntas por medio de un dispositivo móvil. Para su funcionamiento se requiere Internet y un móvil o tableta.

En la tabla 1 se presenta una comparativa de las características de cada una de las herramientas. Ambas herramientas son gratuitas para los usuarios, por lo que estos no tienen la obligación de descargar la aplicación para crear el test o responder a las preguntas planteadas por el profesor. El acceso al cuestionario por parte del alumno se realiza mediante un código, siendo en el caso de Socrative siempre el mismo (creado por el profesor) y distinto en el caso de Kahoot! (es la propia herramienta la que da el código, siempre numérico, cada vez que se lanza el cuestionario). En cuanto a la participación en el test, el alumno ha de registrarse al momento de participar en la actividad; señalando el número total de participantes en el caso de Kahoot!, pero no en Socrative; aunque en este último la identidad puede ocultarse. Para elaborar la actividad no existe un límite de preguntas. Pero sí de opciones de respuesta en el caso de Kahoot!, ya que estas deben ser siempre 4, entretanto las preguntas en Socrative no están limitadas. La tipología de las preguntas pueden ser en el caso de Socrative de 3 tipos: tipo test, verdadero/falso y respuesta corta, sin limitación de caracteres; mientras que en Kahoot!, solo puede ser de tipo test (limitadas las preguntas a 95 caracteres y a 60 las respuestas). En ambos casos se puede añadir una imagen; aunque Kahoot!, permite añadir un vídeo. Al lanzar el cuestionario, en ambos casos, el profesor decide si el alumno responde a su propio ritmo las preguntas o si todos contestan a la pregunta que ha establecido en ese instante; permitiendo en el caso de Kahoot!, el establecer un tiempo de respuesta o en el caso de Socrative plantear en el momento una pregunta de forma rápida. Al finalizar la sesión se pueden 
elaborar cuestionarios para comprobar en el momento lo aprendido y valorarla.

El feedback de las respuestas es inmediato: con Socrative dependerá de la modalidad escogida por el profesor y con Kahoot!, un ranking con los 5 primeros participantes dado que se valora tanto la superación de la pregunta como la rapidez de la respuesta. Se pueden crear equipos de forma aleatoria en el caso de Socrative, pero no de forma voluntaria; mientras que no existe esta opción en Kahoot! En ambos casos se genera un informe con los resultados de la sesión, indicando los nombres y la puntuación de cada participante.

Tabla 1. Comparativa entre las HR

\begin{tabular}{|c|c|c|}
\hline & Socrative & Kahoot! \\
\hline Acceso gratuito & SI & SI \\
\hline Obligatoriedad de descarga de la aplicación & NO & NO \\
\hline Doble plataforma: para el profesor y el alumno & $\mathrm{SI}$ & $\mathrm{SI}$ \\
\hline Acceso con código & Siempre el mismo & Cambiante \\
\hline Identidad del participante & $\mathrm{SI}$ & $\mathrm{SI}$ \\
\hline Anonimato de participantes & $\mathrm{SI}$ & NO \\
\hline Señala el número de participantes & NO & $\mathrm{SI}$ \\
\hline Descarga test en papel & $\mathrm{SI}$ & NO \\
\hline $\begin{array}{l}\text { Descarga informe con preguntas y respuestas } \\
\text { individuales }\end{array}$ & $\mathrm{SI}$ & $\mathrm{SI}$ \\
\hline Limitación número de preguntas & NO & NO \\
\hline Opciones de respuesta & Ilimitadas & 4 \\
\hline Establecimiento tiempo de respuesta & NO & SI \\
\hline Música & NO & $\mathrm{SI}$ \\
\hline Limitación de caracteres en la pregunta & NO & $\mathrm{SI}$ \\
\hline Tipología de preguntas diferentes & 3 & 1 \\
\hline Inclusión de imágenes & $\mathrm{SI}$ & $\mathrm{SI}$ \\
\hline Inclusión de vídeos & NO & SI \\
\hline Feedback inmediato & $\mathrm{SI}$ & $\mathrm{SI}$ \\
\hline Profesor puede moderar avance de las preguntas & $\mathrm{SI}$ & $\mathrm{SI}$ \\
\hline Creación de equipos aleatorios & SI & NO \\
\hline Creación de equipos voluntarios & NO & NO \\
\hline $\begin{array}{l}\text { Facilita interacción con el profesor de forma } \\
\text { inmediata }\end{array}$ & NO & $\mathrm{SI}$ \\
\hline Ranking por aciertos/tiempo & NO & SI \\
\hline
\end{tabular}

Fuente: elaboración propia por parte de las autoras.

\section{Metodología}

\section{Diseño}

Este estudio se realizó en el segundo semestre del curso 2016-2017 en una universidad pública española, concretamente en la Universitat Jaume I, y en la asignatura optativa "Marketing en el punto de venta" de último curso que se imparte en dos titulaciones distintas: en el Grado de Publicidad y Relaciones Públicas (código PU0944) y en el Grado de Comunicación Audiovisual (CA0944); teniendo en ambos casos el mismo contenido, facilitando de esta forma el análisis comparativo de las dos HRA. Para los alumnos de Publicidad y Relaciones Públicas se utilizó la herramienta Socrative; mientras que para los alumnos de Comunicación Audiovisual fue Kahoot! 
Esta investigación se desarrolló con la finalidad de examinar la influencia de la utilización de las nuevas tecnologías en las aulas universitarias con el objetivo de saber si estas herramientas tecnológicas son motivadoras, fomentadoras de la participación del alumnado y facilitadoras del aprendizaje y al mismo tiempo saber la opinión de los estudiantes sobre la funcionalidad de estas herramientas tecnológicas para futuras implementaciones.

Al inicio del curso se procedió a realizar un test inicial para saber qué conocimientos tenían los alumnos referentes a la asignatura. La teoría de la asignatura se divide en seis unidades teóricas, y al finalizar cada unidad (en la clase siguiente antes de empezar la nueva sesión) los estudiantes debían responder diez preguntas tipo test de múltiple respuesta; aunque con una única respuesta correcta referida a la sesión anterior. Los alumnos obtenían un feedback inmediato, comprobando al momento si habían retenido los conocimientos más relevantes vistos en la clase teórica previa. Al final del curso se volvió a pasar el test inicial a los alumnos para evidenciar que realmente habían aprendido durante la impartición de la asignatura. El último día de la asignatura, los alumnos respondieron a un cuestionario sobre la satisfacción del uso de estas herramientas para facilitar el aprendizaje en el aula. Por tanto, el diseño de este estudio se llevó a cabo en tres pasos: (i) el profesor creó el test inicial (y final) junto con los 6 test (uno para cada sesión teórica), tanto para Socrative como para Kahoot!, además explicó a los estudiantes la metodología de la asignatura; (ii) se realizaron seis test para cada HRA, al finalizar cada sesión los estudiantes tenían que responder diez preguntas tipo test de múltiple respuesta basadas en la última sesión teórica cuya nota individual era la media de los test obtenidos por los integrantes del grupo (de 4 personas). Los test eran individuales y como consecuencia los estudiantes no podían compartir la información y las respuestas con otros estudiantes. Estas herramientas permiten al profesor saber si los alumnos han comprendido los conocimientos de la sesión teórica anterior antes de empezar una unidad nueva. Para incrementar la motivación de los estudiantes, dependiendo de los resultados obtenidos en los test, estos podían obtener hasta un punto adicional de la nota final; (iii) se les pasó un cuestionario de opinión sobre la actividad realizada.

\section{Muestra}

La base de datos para este estudio incluyó los alumnos de la asignatura "Marketing en el punto de venta", de dos titulaciones de la Universitat Jaume I, durante el segundo semestre del curso académico 2016-2017.

La muestra estuvo compuesta por 60 estudiantes, de los cuales 41 correspondían al Grado de Publicidad y Relaciones Públicas y 19 al Grado de Comunicación Audiovisual. Tal y como se observa en la tabla 2, en ambos grados, el porcentaje de mujeres es bastante superior al de los hombres. En concreto, en Publicidad y Relaciones Públicas había 30 mujeres (73,1\%) y 11 hombres (26,9\%). En lo que respecta a Comunicación Audiovisual, 16 eran mujeres $(84,2 \%)$ y 3 hombres $(15,8 \%)$.

Tabla 2. Descripción de la muestra

\begin{tabular}{lcc}
\hline & Publicidad y Relaciones Públicas & Comunicación Audiovisual \\
\hline Mujeres & 30 & 16 \\
\hline Hombres & 11 & 3 \\
\hline Total & 41 & 19 \\
\hline
\end{tabular}

Fuente: elaboración propia por parte de las autoras.

\section{Resultados}

Los cuestionarios planteados para ambas asignaturas eran los mismos. Aunque en la herramienta Kahoot!, tenía un tiempo limitado de 20 segundos; mientras que en Socrative no había tiempo límite de respuesta, por lo que los estudiantes contestaban sin esta presión.

El primer día de clase los alumnos respondieron un test inicial sobre conocimientos básicos de la asignatura, obteniendo en ambos casos una 
media por debajo del cinco sobre diez. En cambio, al finalizar la asignatura se volvió a pasar el mismo test con unos resultados muy favorables; en donde el de la asignatura con la aplicación Kahoot!, fue superior (nueve puntos sobre diez) (figuras 1, 2).

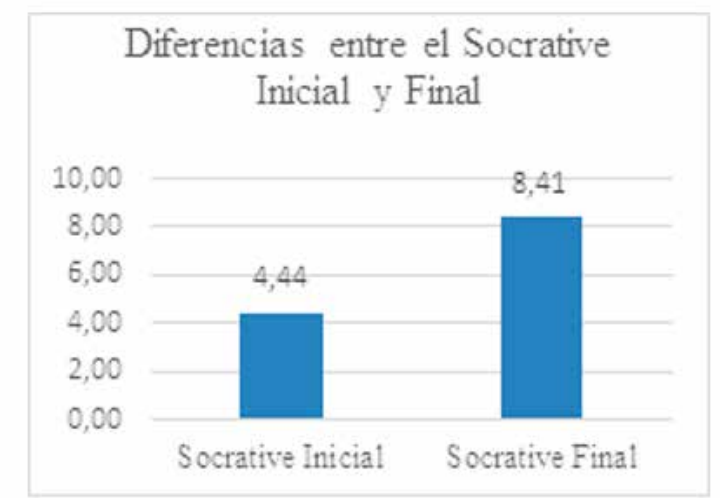

Figura 1. Diferencia entre Socrative, inicial y final. Fuente: elaboración propia por parte de las autoras.

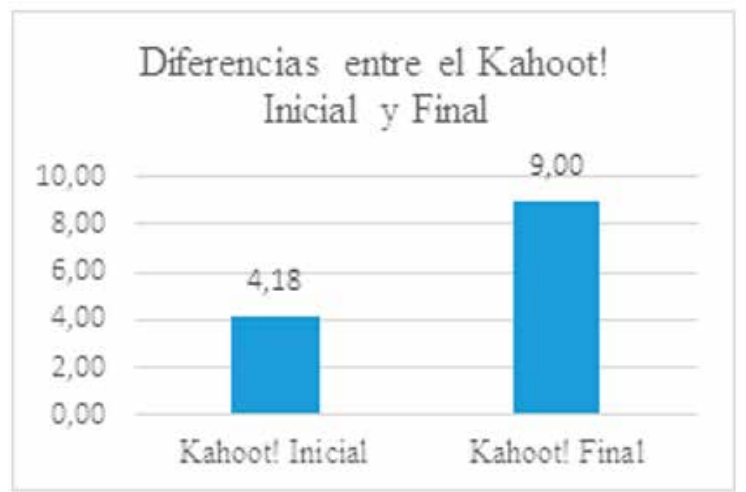

Figura 2. Diferencia entre Kahoot!, inicial y final. Fuente: elaboración propia por parte de las autoras.

En las figuras 3 y 4 se detalla la nota media de los 6 test que realizaron los alumnos durante el curso y que daban opción a conseguir hasta un punto extra en la asignatura. Es fundamental incidir que la nota de toda la asignatura fue grupal, incluyendo los resultados de las HRA, por lo que si algún estudiante no acudía a clase la media de su equipo bajaba considerablemente. Es por este motivo, junto con el tiempo limitado para el Kahoot!, donde se observa una clara diferencia en los resultados. Así pues, con la HRA Kahoot!, se consiguió la media en torno al medio punto; mientras con Socrative se acerca al 0,8 punto.

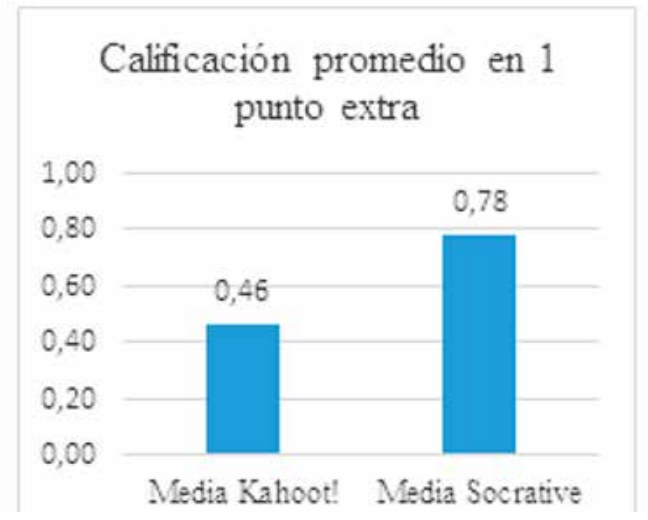

Figura 3. Nota media de los 6 test realizados durante el semestre. Fuente: elaboración propia por parte de las autoras.

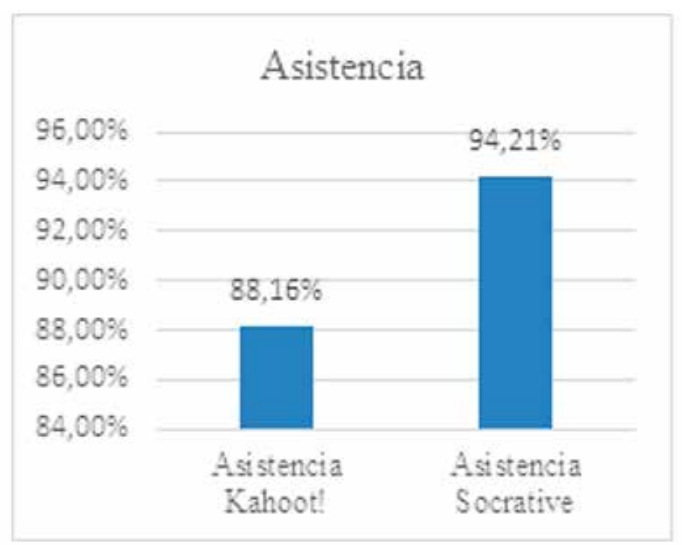

Figura 4. Asistencia a clase. Fuente: elaboración propia por parte de las autoras.

Como se ha destacado en las figuras anteriores, las notas medias de los test y la asistencia a las clases fueron superiores para la herramienta Socrative que para Kahoot! Esta diferencia también se aprecia en la nota final de la asignatura (que incluye los 6 test y el examen final), siendo de un cuarto de punto superior la de Socrative sobre Kahoot! (figura 6); sin embargo la nota del examen final (sin los test del semestre) fue superior, aunque por pocas centésimas, en la asignatura donde se utilizó la herramienta Kahoot! (figura 5). En este sentido el resultado final en ambas titulaciones es más que satisfactorio, consiguiendo que el $100 \%$ del alumnado superase la asignatura. En línea con los resultados obtenidos en Lim (2017), la utilización de un modelo interactivo en el proceso de enseñanza-aprendizaje mejora la experiencia y los resultados académicos obtenidos por los alumnos. 


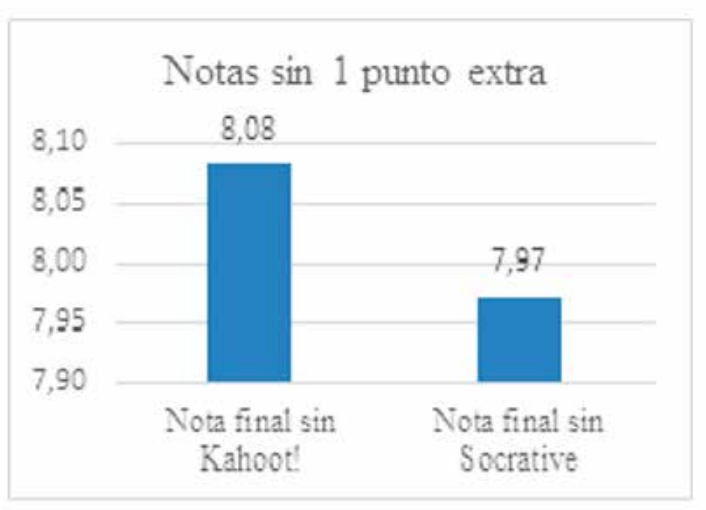

Figura 5. Notas finales del examen de la asignatura. Fuente: elaboración propia por parte de las autoras.

Al finalizar el semestre, los alumnos respondieron un cuestionario sobre satisfacción del uso de las herramientas utilizadas en las dos titulaciones. En relación a la opinión de los estudiantes sobre la funcionalidad de estas herramientas, los

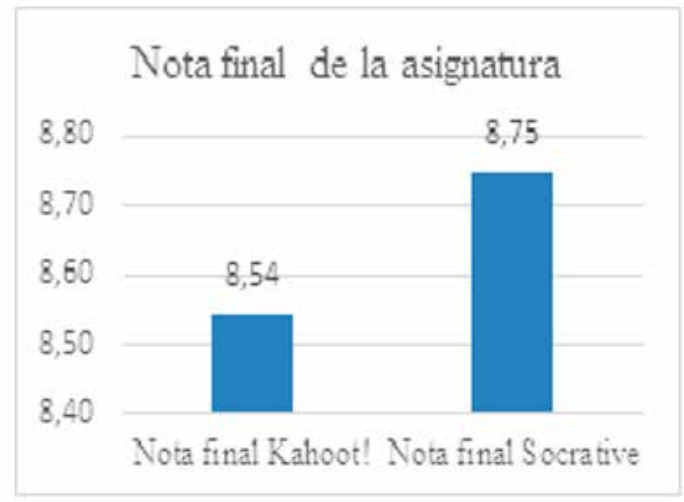

Figura 6. Notas finales de la asignatura (incluyendo los 6 test y el examen final) en las diferentes HRA. Fuente: elaboración propia por parte de las autoras.

alumnos de ambas asignaturas consideraron que eran herramientas adecuadas; aunque los estudiantes que utilizaron la herramienta Socrative, la consideraban indiferente en un $15 \%$ (figura 7).

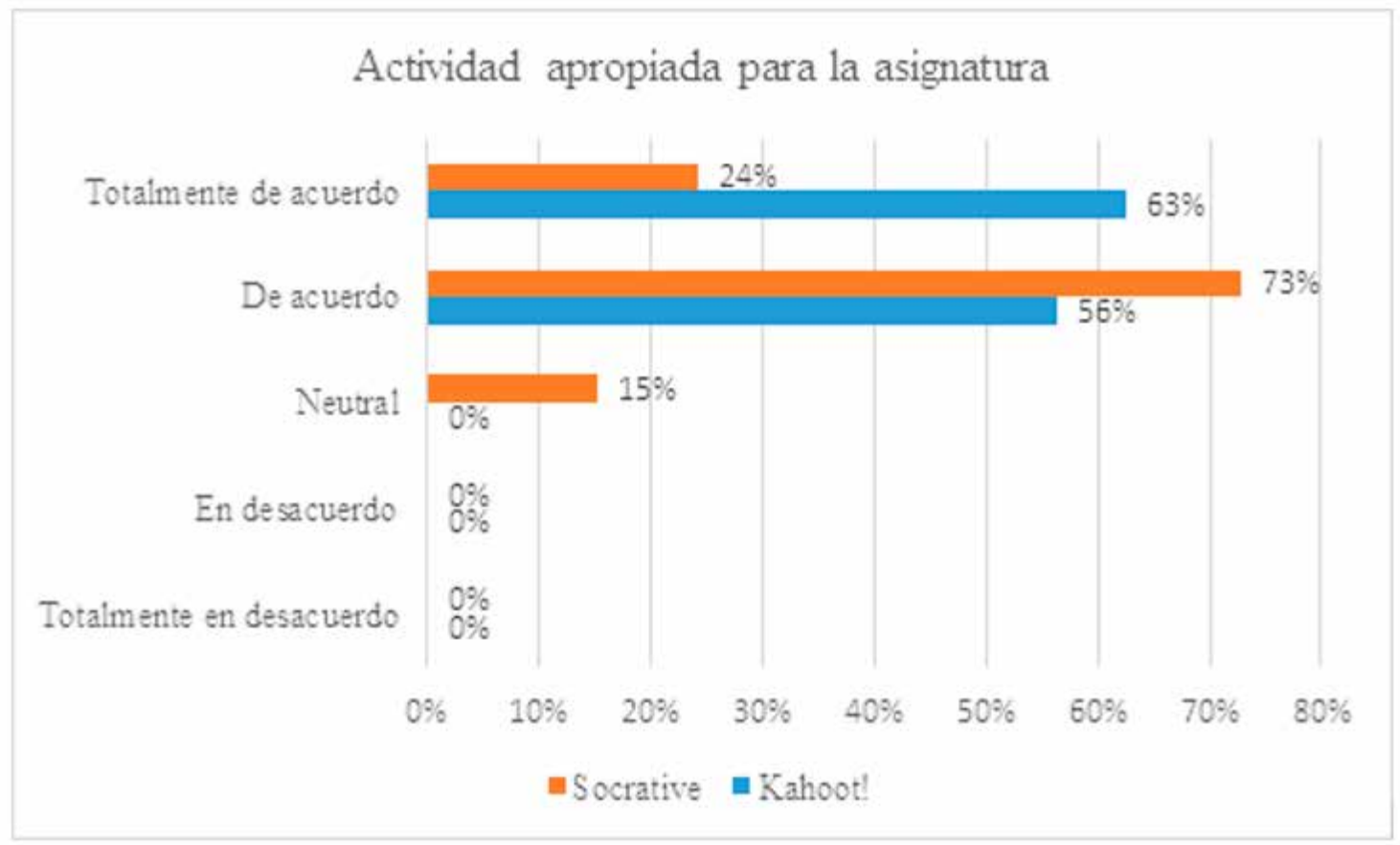

Figura 7. Utilidad de la actividad para la asignatura. Fuente: elaboración propia por parte de las autoras. 
La figura 8, muestra la satisfacción de la HRA con el aprendizaje de la asignatura. En torno al $90 \%$ de los alumnos que han utilizado Kahoot!, están satisfechos o muy satisfechos (Iwamoto et al., 2017); mientras que la cifra baja hasta el 50 \% en los estudiantes que utilizaron Socrative (ParraSantos et al., 2017).

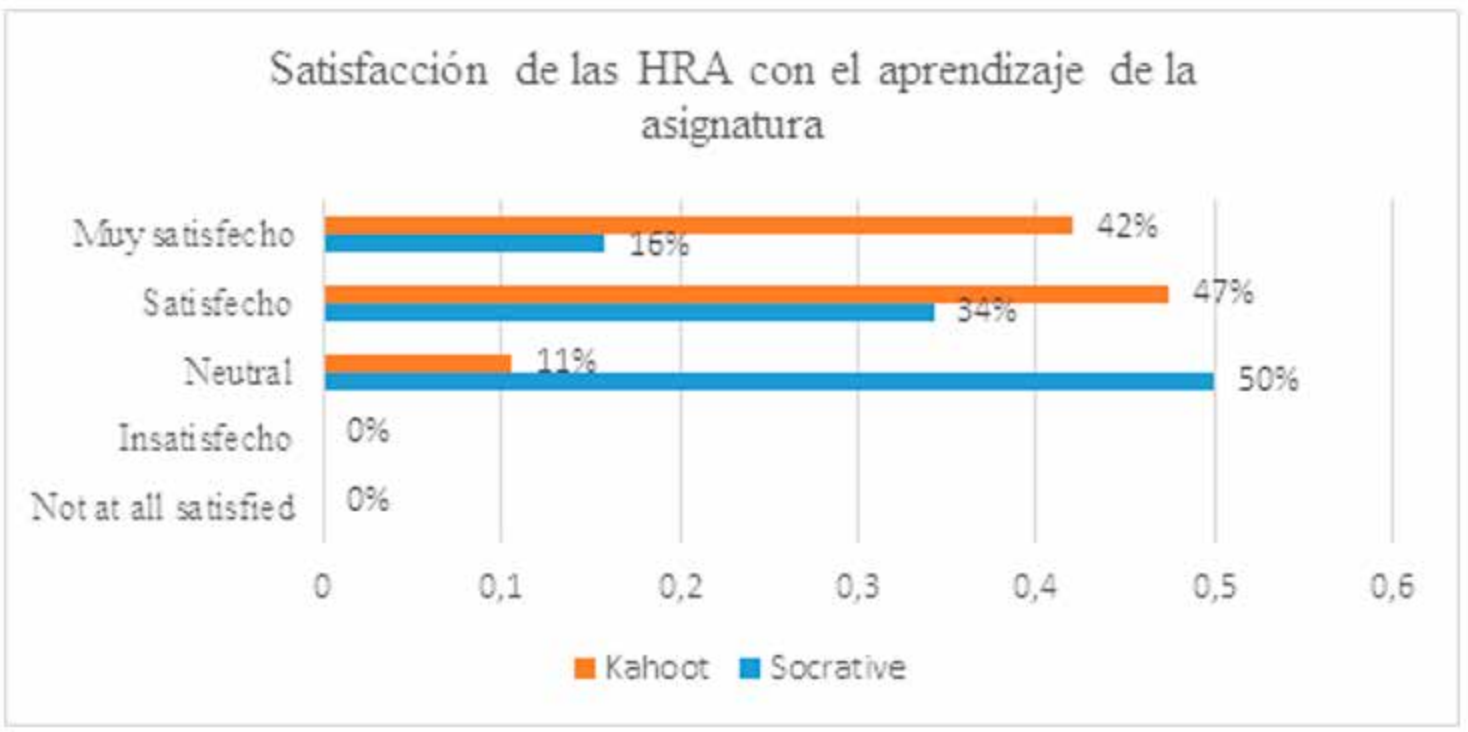

Figura 8. Satisfacción de la HRA con el aprendizaje de la asignatura. Fuente: elaboración propia por parte de las autoras.

Por último, la figura 9 destaca que más del $90 \%$ de los alumnos de ambas asignaturas consideran la actividad desarrollada por la HRA útil para su futuro; siendo del $100 \%$ en el caso de los alumnos que utilizaron Kahoot! $\mathrm{Al}$ igual que en el estudio realizado por Al-Emran et al. (2016), los alumnos recomendarían estas herramientas tecnológicas para su utilización en actividades futuras dado que consideran al m-learning como una tecnología pedagógica prometedora para emplear en entornos educativos superiores.

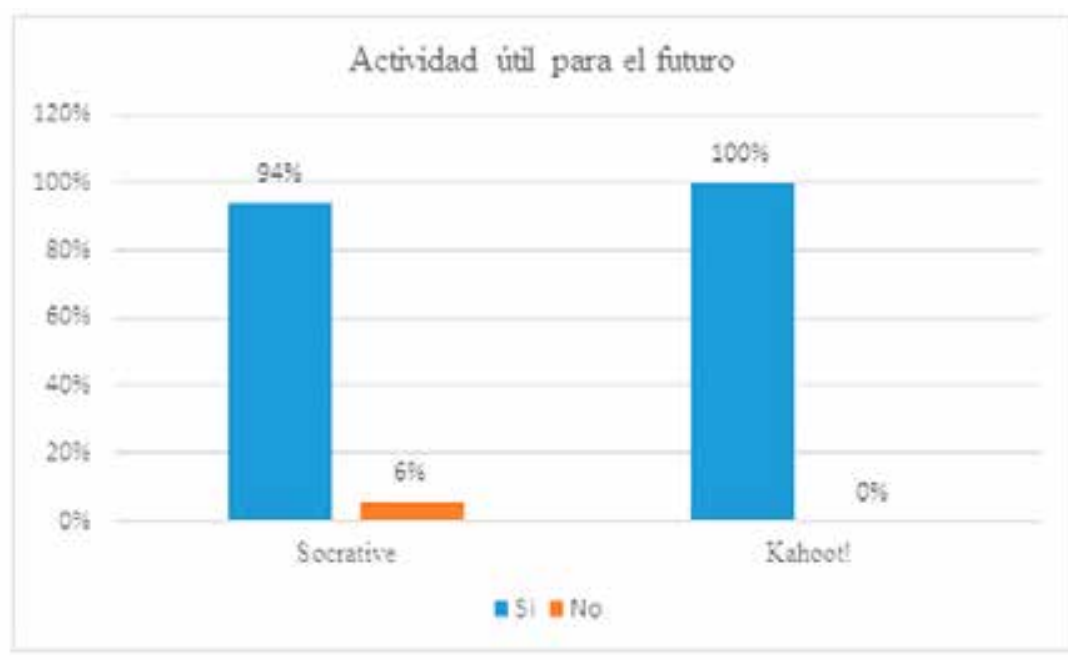

Figura 9. Actividad útil para el futuro. Fuente: elaboración propia por parte de las autoras. 
Los resultados obtenidos en este estudio indican que los alumnos consideran la utilización de HRA (concretamente Socrative y Kahoot!) como útiles para el proceso de enseñanza-aprendizaje y que es necesaria su implantación en otras asignaturas y titulaciones.

\section{Conclusiones}

Con este trabajo hemos aplicado dos HRA: Socrative y Kahoot!, en dos asignaturas distintas en una universidad pública española. Se deseaba analizar si el uso de las HRA conseguía mejorar la participación y motivación de los estudiantes y facilitaba el aprendizaje de la asignatura. Las conclusiones a las que se llegan son las siguientes.

En primer lugar, la asistencia a clase ha sido superior al $88 \%$; bastante mayor que la media de asistencia en la universidad.

En segundo lugar, el uso de las HRA ha proporcionado una mejora notable en el aprendizaje (medido en los resultados obtenidos desde la realización del cuestionario inicial hasta el final por parte de los alumnos de las dos titulaciones); así como en el rendimiento final de las asignaturas con notas superiores al notable de media en los exámenes finales. Esto está en línea con los resultados obtenidos por Hamdan y Amorri (2016), quienes informaron que el uso del m-learning en clase puede mejorar el proceso de aprendizaje.

En tercer lugar, se observan diferencias entre los dos instrumentos tanto en la asistencia como en el aprendizaje. Socrative consiguió una mayor asistencia a clase; aunque Kahoot!, mostró mejor rendimiento en el examen final y mayor aprendizaje desde el cuestionario inicial al final.

En cuarto lugar, la motivación de los estudiantes se puede medir por el grado de utilidad y satisfacción que les proporciona el uso de estos instrumentos. Los alumnos consideraron la utilización de HRA (en más de un $94 \%$ ) útil para su futuro, útil para el aprendizaje de la asignatura (en un $80 \%$ ) y estando satisfechos con su uso en la asignatura (50\%). No obstante, un $15 \%$ de los estudiantes se mostraron indiferentes con el uso de estas herramientas tecnológicas en el aula. Este último hecho puede explicarse porque a medida que pasa el tiempo, la dificultad de las asignaturas es superior al atractivo que tienen las HRA (Balta and Tzafilkou, 2019).

Por último, también se detectaron diferencias entre las dos herramientas. Los usuarios de Kahoot!, la consideraron más útil para su futuro y para el aprendizaje de la asignatura; estando más satisfechos que los usuarios de Socrative. Para los alumnos, Kahoot!, es visto más como un juego (con más espíritu competitivo) que Socrative; pues el segundo, es considerado más como la realización de múltiples exámenes. Esto nos podría indicar que sería necesario incluir estos resultados en el diseño de la metodología a aplicar en futuras asignaturas.

Estos resultados, creemos, pueden tener diversas implicaciones para debates centrados en la educación. Así, los resultados obtenidos serían útiles para profesores universitarios cuando diseñan las metodologías de las asignaturas que imparten. En particular, podrían incluir actividades que utilizan HRA para incentivar la asistencia a las clases teóricas y mejorar el rendimiento académico de los alumnos. Sin embargo la implicación más importante de este estudio es que en el sistema de educación hay un gran número de asignaturas que contienen gran variedad de conceptos específicos -aunque no incluyen competencias necesariasque todos los alumnos deberían adquirir para su desarrollo personal, académico y profesional (Dervan, 2014). Por ello estos resultados deberían incentivar a los responsables en educación a desarrollar programas de estudio que incluyan el desarrollo de competencias utilizando HRA (como Socrative y Kahoot!) en las aulas o trabajos en equipo. Otra implicación que se deriva de este estudio es que el uso de las nuevas tecnologías en las aulas mejora el proceso de enseñanza-aprendizaje dado que este proceso puede ser personalizado para cada estudiante, puede ayudar a los alumnos que tengan dificultades o puede mejorar e incrementar el aprendizaje de aquellos alumnos que quieren saber más de la materia.

Los hallazgos de este estudio están en consonancia con los resultados de la literatura previa de varias maneras: (i) estos hallazgos apoyan la hipótesis de que el Smartphone es considerado una herramienta esencial para la educación superior (Yu, 2012); (ii) el sistema m-learning motiva 
a los alumnos a asistir a clase e incrementa sus notas finales, por lo tanto la repercusión del uso de las nuevas tecnologías en las aulas universitarias tomará una nueva perspectiva dado que esta tecnología puede ser un complemento a la educación tradicional (Laouris and Eteokleous, 2005) o un mecanismo para conectar las áreas formales e informales de la educación superior (Merchant, 2012). Por esta razón, este estudio puede ser considerado una herramienta para explicar la importancia de las nuevas tecnologías en las aulas universitarias.

Por otra parte, somos conscientes de las limitaciones que presenta este estudio y que se pueden concretar en las siguientes: (i) la elevada dependencia de la red de datos, ya que sin una buena conexión a Internet la actividad no se puede realizar; (ii) los profesores deben tener unos conocimientos básicos sobre el uso de estas herramientas y metodologías que utilizan Internet, así como estar abiertos a cambios en la metodología tradicional; (iii) los resultados obtenidos son por equipos y no individuales, por lo que hay que tener especial cuidado en el momento de interpretarlos dado que si un integrante del grupo no se esfuerza en el estudio del temario la nota del grupo se ve reducida. Estos resultados están en la línea de los obtenidos por Balta y Tzafilkou (2019), quienes documentaron que los alumnos prefieren trabajar de forma individual a la grupal en el uso de HRA; (iv) es posible que existan factores no considerados y que afecten a nuestra variable dependiente.

Para finalizar, como futuras líneas de investigación que ampliarían el alcance de este trabajo, podemos destacar la conveniencia de examinar el uso de estas herramientas tecnológicas en asignaturas obligatorias donde hay aproximadamente 250 estudiantes para poder confirmar los resultados obtenidos. Por otra parte, aportaría mayor valor al estudio analizar qué teorías se utilizan para la realización de los materiales utilizados en el proceso de enseñanza-aprendizaje con herramientas tecnológicas.

\section{Referencias}

Al-Emran, M. et al. (2016). Investigating attitudes towards the use of mobile learning in higher education. Computers in Human Behavior, 56, 93-102.

Ally, M. and Prieto-Blázquez, J. (2014). What is the future of mobile learning in education? RUSC. Universities and Knowledge Society Journal, 11 (1), 142-151.

Balta, N. and Tzafilkou, K. (2019). Using Socrative software for instant formative feedback in physics courses. Education and Information Technologies, 24 (1), 307-323.

Bicen, H. and Kocakoyun, S. (2018). Perceptions of students for gamification approach: Kahoot as a case study. International Journal of Emerging Technologies in Learning, 13 (02), 72-93.

Brebera, P. (2016). Ways of enriching foreign language courses at the university: Digital badges and mobile learning. Recuperado de https://library.iated.org/ view/BREBERA2016WAY.

Crompton, H. (2013). A historical overview of mobile learning: Toward learner-centered education. En Berge, Z.L. and Muilenburg, L.Y. (Ed.), Handbook of mobile learning (pp. 3-14). New York, USA: Routledge.

Dervan, P. (2014). Increasing in-class student engagement using Socrative (an online Student Response System). AISHE-J: The All Ireland Journal of Teaching and Learning in Higher Education, 6 (3), 1801-18013.

Fundación Telefónica. (2014). Mi móvil al servicio de la comunidad: aprender y compartir. Recuperado de https://observatorio.profuturo.education/laboratorio-m-learning/mi-movil-al-servicio-de-la-comunidad-aprender-y-compartir/.

Hamdan, K. and Amorri, A. (2016). Mobile learning devices to assist dyslexic students to improve their English reading proficiency. Recuperado de https://library.iated.org/view/HAMDAN2016MOB.

Herrington, A. (2009). Using a smartphone to create digital teaching episodes as resources in adult education. En Herrington, J. et al. (Ed.), New technologies, new pedagogies: Mobile learning in higher education. Wollongong, Australia: University of Wollongong. 
Humnante-Ramos, P. and García-Peñalvo, F.J. (2013). Contribution of virtual classrooms to the personal learning environments (PLE) of the students of the career of informatics applied to education of national university of Chimborazo. Recuperado de https://dl.acm.org/citation.cfm?id=2536614.

Iwamoto, D.H. et al. (2017). Analyzing the Efficacy of the Testing Effect Using Kahoot ${ }^{\mathrm{TM}}$ on Student Performance. Turkish Online Journal of Distance Education, 18 (2), 80-93.

Kapp, K.M. (2012). The Gamification of Learning and Instruction: Case-Based Methods and Strategies for Training and Education. New York, USA: John Wiley \& Sons.

Laouris, Y. and Eteokleous, N. (2005). We need an educationally relevant definition of mobile learning. Recuperado de https://www.semanticscholar. org/paper/WE-NEED-AN-EDUCATIONALLY-RELEVANT-DEFINITION-OF-Laouris/a42110ce607e186a433455f32ec54ba8032878f1.

Lim, W.N. (2017). Improving student engagement in higher education through mobile-based interactive teaching model using socrative. Recuperado de https://ieeexplore.ieee.org/document/7942879.

Masrom, M. and Ismail, Z. (2010). Benefits and barriers to the use of mobile learning in education: Review of literature. En Guy, R. (Ed.), Mobile learning: Pilot projects and initiatives (pp. 9-26). California, USA: Informing Science Press.

Merchant, G. (2012). Mobile practices in everyday life: Popular digital technologies and schooling revisited. British Journal of Educational Technology, 43 (5), 770-782.

Mtebe, J. and Raisamo, R. (2014). Investigating students' behavioural intention to adopt and use mobile learning in higher education in East Africa. International Journal of Education and Development using ICT, 10 (3), 4-20.

Naismith, L. et al. (2004). Literature Review in Mobile Technologies and Learning. Bristol, England: Futurelab.

Parra-Santos, M.T. et al. (2017). La aplicación SOCRATIVE como herramienta de evaluación y precursor de la participación en el aula. En Roig-Vila, R. (Ed.), Investigación en docencia universitaria. Diseñando el futuro a partir de la innovación educativa (pp. 677-683). Barcelona, España: Octaedro.
Paz-Albo Prieto, J. (2014). El impacto de los dispositivos móviles como sistemas de respuesta personal en la enseñanza de futuros maestros: un estudio de caso. El Guiniguada. Revista de investigaciones y experiencias en Ciencias de la Educación, 23, 125133.

Sánchez-Prieto, J.C. et al. (2014). Understanding mobile learning: Devices, pedagogical implications and research lines. Revista Teoría de la Educación: Educación y Cultura en la Sociedad de la Información, 15 (1), 20-42.

Suñol, J.J. et al. (2016). Physics with mobile technology. En I Jornadas de innovación DIMEU. Dispositivos Móviles en la Educación Universitaria, Valencia, España.

UNESCO. (2016). Mobile Learning. Recuperado de https://en.unesco.org/news/unesco-mobile-learning-week-2016-innovation-quality-education.

Vázquez-Cano, E. (2014). Mobile distance learning with smartphones and apps in higher education. Educational Sciences: Theory and Practice, 14 (4), 1505-1520.

Yu, F. (2012). Mobile/Smartphone Use in Higher Education. Recuperado de http://swdsi.org/swdsi2012/ proceedings_2012/papers/Papers/PA144.pdf.

Yu, F. and Conway, A.R. (2012). Mobile/smartphone use in higher education. Recuperado de http://swdsi. org/swdsi2012/proceedings_2012/papers/Papers/PA144.pdf.

West, M. (2012). Turning on Mobile Learning: Global Themes. Recuperado de https://unesdoc.unesco. org/ark:/48223/pf0000216451. 